\title{
EVALUATION OF HAND FUNCTIONALITY, DISABILITY AND QUALITY OF LIFE IN PATIENTS WITH SYSTEMIC SCLEROSIS: A CROSS-SECTIONAL STUDY
}

Amanda Maria da Silva (Universidade Federal de Pernambuco, Recife, PE, Brasil), Letticia Cristina Santos

Cardozo Roque (Universidade Federal de Pernambuco, Recife, PE, Brasil), Angélica da Silva Tenório

(Universidade Federal de Pernambuco, Recife, PE, Brasil), Daniel Neri da Matta (Universidade Federal de Pernambuco, Recife, PE, Brasil), Rafaela Silva Guimarães Gonçalves (Universidade Federal de Pernambuco, Recife, PE, Brasil), Lilian David de Azevedo Valadares (Universidade Federal de Pernambuco, Recife, PE, Brasil), Andréa Tavares Dantas (Universidade Federal de Pernambuco, Recife, PE, Brasil), Angela Luzia Branco Pinto Duarte (Universidade Federal de Pernambuco, Recife, PE, Brasil)

\section{BACKGROUND}

Hand involvement due to cutaneous, vascular and joint changes in systemic sclerosis (SSc) patients is one of the main causes of disability attributed to the disease and is associated with a significant decrease in quality of life. The characterization of these commitments contributes to the formulation of more effective treatment strategies. The objective of the present study was to evaluate the functionality of hands, disability and quality of life in SSc patients, to compare the differences between clinical forms and to verify the association with clinical manifestations of the disease.

\section{MATERIALS AND METHODS}

Cross-sectional, descriptive and analytical study. The sample comprised 60 patients classified as SSc according to the criteria of the ACR / EULAR 2013. The functionality of the hands was evaluated through Cochin Hand Functional Scale (CHFS), the quality of life by the 12-Item Short-Form Health Survey - PCS physical component summary (SF-12PCS), and MCS-mental component summary (SF-12 MCS) and the disability by the Scleroderma Health Assessment Questionnaire (SHAQ).

\section{RESULTS}

Most of the patients were female (91.7\%), with a mean age of $47.1 \pm 12.9$ years old and $53.3 \%$ had a limited cutaneous form. The mean results for CHFS, SHAQ, SF-12 PCS and SF-12 MCS were 14.5 (6.0$29.75), 1.01 \pm 0.56,35.04 \pm 8.09,40.94 \pm 10.56$, respectively. In the comparison of clinical forms, PCS-SF 12 showed a better result in the diffuse form of the disease $(p=0.04)$; there were no differences regarding the functionality, disability and mental component of quality of life. There were no associations between the CHFS, SHAQ and SF-12 scores and clinical manifestations of the disease. A moderate correlation was observed between CHFS and SHAQ $(r=0.66, p<0.0001)$ and weak correlation between CHFS and SF-12 PCS $(r=-0.31, p=0.02)$ and CHFS and SF- $12 \operatorname{MCS}(r=-0.28, p=0.03)$.

\section{CONCLUSION}

Patients with SSc present an important impairment of hand function, quality of life and functional capacity, and those with limited cutaneous form presented worse scores of the physical component in the evaluation of quality of life. 